\title{
Hippocampal spine changes across the sleep-wake cycle: corticosterone and kinases
}

\author{
Muneki Ikeda', Yasushi Hojo',2, Yoshimasa Komatsuzaki', Masahiro Okamoto,,3, \\ Asami Kato', Taishi Takeda' and Suguru Kawato ${ }^{1,2,4}$ \\ ${ }^{1}$ Department of Biophysics and Life Sciences, Graduate School of Arts and Sciences, University of Tokyo, \\ 3-8-1 Komaba, Meguro-ku, Tokyo 152-8902, Japan \\ ${ }^{2}$ Bioinformatics Project of Japan Science and Technology Agency, University of Tokyo, Tokyo, Japan \\ ${ }^{3}$ Laboratory of Exercise Biochemistry and Neuroendocrinology, Faculty of Health and Sports Sciences, \\ University of Tsukuba, 1-1-1 Tennodai, Tsukuba, Ibaraki 305-8577, Japan \\ ${ }^{4}$ Department of Urology, Graduate School of Medicine, Juntendo University, 2-1-1 Hongo, Tokyo 113-8424, Japan
}

\author{
Correspondence \\ should be addressed \\ to S Kawato \\ Email \\ kawato@bio.c.u-tokyo.ac.jp
}

\begin{abstract}
The corticosterone (CORT) level changes along the circadian rhythm. Hippocampus is sensitive to CORT, since glucocorticoid receptors are highly expressed. In rat hippocampus fixed in a living state every $3 \mathrm{~h}$, we found that the dendritic spine density of CA1 pyramidal neurons increased upon waking (within $3 \mathrm{~h}$ ), as compared with the spine density in the sleep state. Particularly, the large-head spines increased. The observed change in the spine density may be due to the change in the hippocampal CORT level, since the CORT level at awake state $(\sim 30 \mathrm{nM})$ in cerebrospinal fluid was higher than that at sleep state $(\sim 3 \mathrm{nM})$, as observed from our earlier study. In adrenalectomized (ADX) rats, such a wake-induced increase of the spine density disappeared. S.c. administration of CORT into ADX rats rescued the decreased spine density. By using isolated hippocampal slices, we found that the application of $30 \mathrm{nM}$ CORT increased the spine density within $1 \mathrm{~h}$ and that the spine increase was mediated via PKA, PKC, ERK MAPK, and LIMK signaling pathways. These findings suggest that the moderately rapid increase of the spine density on waking might mainly be caused by the CORT-driven kinase networks.
\end{abstract}

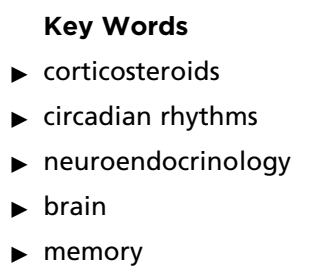

Journal of Endocrinology (2015) 226, M13-M27

\section{Introduction}

Corticosterone (CORT), known as a stress hormone, is released from the adrenal cortex, and the plasma CORT level is regulated by adrenocorticotropic hormone (ACTH) stimulation. ACTH is secreted from the pituitary gland in response to the arrival of the corticotropin-releasing hormone, which is released from the paraventricular hypothalamic nucleus (PVN). Since PVN has direct and indirect projections from the suprachiasmatic nucleus (Buijs et al. 1993, Vrang et al. 1995), the CORT level changes in both the plasma and brain along the circadian rhythm (Migeon et al. 1956, Moore \& Eichler 1972, Qian et al. 2012).
Qian et al. showed the high synchronicity of CORT oscillation between the blood and hippocampus by using microdialysis. Our previous study showed that in the cerebrospinal fluid (CSF), the CORT concentration is $\sim 30 \mathrm{nM}$ in the awake state and $\sim 3 \mathrm{nM}$ in the sleep state (Higo et al. 2011). These concentrations are much lower than the stress level of CORT $(\sim 1 \mu \mathrm{M})$, which causes neural atrophy and memory impairments (Woolley et al. 1990, Krugers et al. 1997). Compared to the stress level of CORT $(\sim 1 \mu \mathrm{M})$, the physiological functions from the lower level CORT $(\sim 30 \mathrm{nM})$ almost remain unraveled. We also do not

Published by Bioscientifica Ltd 
know the role of the diurnal rise and fall of such a low level CORT in the brain.

The hippocampus, the center for learning and memory, is particularly sensitive to CORT (Kim et al. 2006, Maggio \& Segal 2010) because glucocorticoid receptors (GR) are abundantly expressed in the hippocampus (Chao et al. 1989, Morimoto et al. 1996). The hypothalamicpituitary-adrenocortical axis (mentioned above) receives feedback regulation from the hippocampus (Sapolsky et al. 1984, Jacobson \& Sapolsky 1991). Recent studies show that the performance of hippocampal-dependent learning and memory fluctuates along the circadian rhythm. The scores on the novel-object recognition task are higher during the dark phase (awake state) than during the light phase (sleep state) (Ruby et al. 2008). The performance of the motor skill learning (cortex-dependent) is also better in the awake state (Liston et al. 2013), and this diurnal change has a strong relationship with the circadian CORT oscillation. Liston et al. demonstrated that the spine (postsynaptic structure) formation of pyramidal neurons in the motor cortex is enhanced by circadian CORT peaks. The spinogenesis of hippocampal pyramidal neurons is also induced by the treatment of CORT on isolated hippocampal slices (Komatsuzaki et al. 2012, Yoshiya et al. 2013). In these studies, however, 200-10 $000 \mathrm{nM} \mathrm{CORT} \mathrm{is} \mathrm{administrated} \mathrm{to}$ increase spines, which is a much higher level than the CORT level in CSF during the circadian CORT peaks ( $30 \mathrm{nM})$. Therefore, the effect from the lower physiological level of CORT on spines should be revealed.

In the current study, we investigated whether the spine density in the CA1 region of the hippocampus changes along the diurnal sleep-wake cycle and whether the circadian rhythm of CORT causes it. We also examined the effect by the treatment of $30 \mathrm{nM}$ CORT on the spine density in the isolated hippocampal slices. The time course of the CORT effect and its signaling cascade within the downstream of the synaptic GR were investigated. We provided detailed understanding of how the diurnal rise in CORT influences dendritic spines in the hippocampus.

\section{Materials and methods}

\section{Animals}

Young adult male Wistar rats (10-11 weeks old, 280-320 g) were purchased from Tokyo Experimental Animals Supply (Tokyo, Japan). All animals were maintained under a $12 \mathrm{~h}$ light:12 h darkness cycle (lights on at $0800 \mathrm{~h}$, lights off at $2000 \mathrm{~h}$ ) and given free access to food and water. The rats were adapted to light-dark conditions for 1 week before the experiments. The Zeitgeber time 0 (ZT0) was designated as lights on, and ZT12 was designated as lights off. Rats fall asleep around ZTO and wake up around ZT12. The experimental procedure of this research was approved by the Committee for Animal Research of the University of Tokyo.

\section{Chemicals}

Lucifer Yellow, CORT, Metyrapone, RU-486, Spironolactone, U0126, and SP600125 were purchased from SigmaAldrich. Actinomycin D and cycloheximide (CHX) were from Wako Pure Chemical Industries (Osaka, Japan). H-89 was from Biomol (Philadelphia, PA, USA). Chelerythrine and LIM kinase inhibitors were from Calbiochem (Cambridge, MA, USA).

\section{Adrenalectomy and s.c. drug administration}

The adrenal glands from 11-week-old male rats were removed bilaterally under deep anesthesia. The rats were given the time to recover from operations and then placed in cages with $0.9 \%$ saline (for maintaining electrolyte balance) and food. These surgeries were performed 1 week before the experiments.

Metyrapone (50 mg/kg body weight) was dissolved in DMSO and diluted with sesame oil to reach its appropriate concentration, enough to suppress the increase of endogenous CORT (Roozendaal et al. 1996). The final volume was adjusted to $400 \mu$ l. S.c. administration was performed at ZT9, $4 \mathrm{~h}$ before the decapitation. During the injection, the rats were gently handled by the experimenter. Similarly, CORT (1 mg/kg body weight) was injected subcutaneously into adrenalectomized (ADX) rats at ZT11, $2 \mathrm{~h}$ before the decapitation.

\section{Imaging and analysis of dendritic spine density}

Slice preparation (from in vivo fixed hippocampus) Hippocampal slices were prepared from a 12-week-old male rat that was deeply anesthetized and perfused transcardially with PBS $(0.1 \mathrm{M}$ phosphate buffer and $0.14 \mathrm{M} \mathrm{NaCl}, \mathrm{pH} 7.3$ ), followed by a fixative solution of $3.5 \%$ paraformaldehyde. Immediately after decapitation, the brain was removed from the skull and post-fixed with the fixative solution. Hippocampal slices, $400 \mu \mathrm{m}$ thick, were sliced with a vibratome (Dosaka, Kyoto, Japan).

Slice preparation (from isolated hippocampus) Twelveweek-old male rats were deeply anesthetized. Decapitation

Published by Bioscientifica Ltd 
was performed without paraformaldehyde fixation procedures. Immediately after decapitation, the brain was removed from the skull and placed in ice-cold oxygenated $\left(95 \% \mathrm{O}_{2}\right.$ and $5 \% \mathrm{CO}_{2}$ ) artificial CSF (ACSF) containing the following (in $\mathrm{mM}$ ): $124 \mathrm{NaCl}, 5 \mathrm{KCl}, 1.25 \mathrm{NaH}_{2} \mathrm{PO}_{4}$, $2 \mathrm{MgSO}_{4}, 2 \mathrm{CaCl}_{2}, 22 \mathrm{NaHCO}_{3}$, and 10 D-glucose (all from Wako Pure Chemical Industries); $\mathrm{pH}$ was set at 7.4. Hippocampal slices, $400 \mu \mathrm{m}$ thick, were sliced with a vibratome. During this moment, these slices were 'fresh' slices without the ACSF incubation. Slices were then incubated in oxygenated ACSF for $2 \mathrm{~h}$ (slice recovery processes) to obtain the widely referred 'acute slices' (Supplementary Fig. S1, see section on supplementary data given at the end of this article). These 'acute slices' were then incubated at room temperature with CORT and other drugs, including kinase inhibitors. After drug exposure, slices were fixed with $4 \%$ paraformaldehyde at $4{ }^{\circ} \mathrm{C}$ for $4 \mathrm{~h}$.

Current injection of neurons by Lucifer Yellow Neurons within slices were visualized by an injection of Lucifer Yellow under a Nikon E600FN microscope (Tokyo, Japan) equipped with a C2400-79H infrared camera (Hamamatsu Photonics, Shizuoka, Japan) and $40 \times$ water immersion lens (Nikon). A glass electrode was filled with $4 \%$ Lucifer Yellow, which was then injected for 5 min using Axopatch 200B (Axon Instruments, Foster City, CA, USA). With this process, approximately five neurons within a $100-200 \mu \mathrm{m}$ depth from the surface of a slice were injected (Huang et al. 2005, Hanani 2012).

Confocal laser microscopy and spine density analysis The imaging was performed from sequential z-series scans with LSM5 PASCAL confocal microscope (Zeiss, Jena, Germany) at high zoom (3.0) with a $63 \times$ water immersion lens, NA 1.2 (Zeiss). For Lucifer Yellow, the excitation and emission wave lengths were 488 and $515 \mathrm{~nm}$ respectively. For an analysis of spines, a threedimensional image was reconstructed from $\sim 40$ sequential z-series sections for every $0.45 \mu \mathrm{m}$. The applied zoom factor (3.0) yielded 23 pixels $/ 1 \mu \mathrm{m}$. The confocal lateral (XY) resolution was $\sim 0.26 \mu \mathrm{m}$. Confocal images were then deconvoluted using AutoDeblur Software (AutoQuant, Rockville, MD, USA).

The density of spines was analyzed with Spiso-3D (mathematical and automated software calculating geometrical parameters of spines), developed by the Bioinformatics Project of Kawato's group (Mukai et al. 2011). Spiso-3D has an equivalent capacity with Neurolucida (MicroBrightField, Williston, VT, USA), which, however, needs time-consuming manual operation. We analyzed the spines in the CA1 pyramidal neurons, along apical dendrites in stratum radiatum of the dorsal hippocampus. We chose the secondary dendrites that were $100-250 \mu \mathrm{m}$ away from the soma. The spine density was calculated from the number of spines per unit length in the dendrite that had a total length of 50-60 $\mu \mathrm{m}$.

Spine shapes were classified into three categories as follows: i) a small-head spine, whose head diameter is smaller than $0.4 \mu \mathrm{m}$; ii) a middle-head spine, which has 0.4-0.5 $\mu \mathrm{m}$ spine head; and iii) a large-head spine, whose head diameter is larger than $0.5 \mu \mathrm{m}$. These three categories were useful to compare the distribution of spine head diameters in each condition. Because the majority of spines ( $>95 \%$ ) had a distinct head and neck, and stubby spines and filopodium did not contribute much to overall changes, we mainly analyzed spines with distinct heads. While counting the spines in the reconstructed images, the position and verification of spines were identified by the rotation of three-dimensional reconstructions and observation of the images in consecutive single planes.

\section{Mass-spectrometric assay of CORT}

Detailed procedures are described elsewhere (Hojo et al. 2009).

\section{Step 1: purification of CORT from hippocampi with normal} phase HPLC A rat was deeply anesthetized and decapitated. The whole hippocampi was removed and homogenized. To extract steroid metabolites, ethyl acetate:hexane (3:2 vol/vol) was applied to the homogenates, which were then mixed. The mixture was centrifuged at $2500 \mathrm{~g}$, and the organic layer was collected. After evaporation, the extracts were dissolved in $1 \mathrm{ml}$ of $40 \%$ methanol $/ \mathrm{H}_{2} \mathrm{O}$ and applied to a Sep-Pak $\mathrm{C}_{18} 3 \mathrm{cc}$ Vac Cartridge (Waters, Milford, MA, USA). The fraction of CORT was separated using a normal phase HPLC system (Jasco, Tokyo, Japan). A silica gel column (Cosmosil 5SL, Nacalai Tesque, Kyoto, Japan) was used.

\section{Step 2: determination of the concentration for CORT using liquid chromatography-tandem mass spectrometry For} determination of the concentration of CORT, the liquid chromatography-tandem mass spectrometry (LC-MS/MS) system, which consists of the Shimadzu HPLC system and an API-5000 triple-stage quadrupole mass spectrometer (Applied Biosystems), was employed. LC separation was performed on a Cadenza CD- $\mathrm{C}_{18}$ column (Imtakt, Kyoto, Japan). MS analysis was operated with electrospray ionization in positive-ion mode. In multiple reaction monitoring

Published by Bioscientifica Ltd 
mode, the instrument monitored the $m / z$ transition from 347 to 121 for CORT. Here, $m$ and $z$ represent the mass and charge of CORT respectively.

The limit of quantification for CORT was $2 \mathrm{pg} / 0.1 \mathrm{~g}$ of hippocampal tissue (Higo et al. 2011). From the calibration curve using standard CORT dissolved in blank samples, the linearity was observed between 1 and $1000 \mathrm{pg}$ for CORT (Supplementary Fig. S2, see section on supplementary data given at the end of this article).

\section{Statistical analysis}

Data are expressed as mean \pm s.e.m. For analysis of the spine density, we used a one-way ANOVA followed

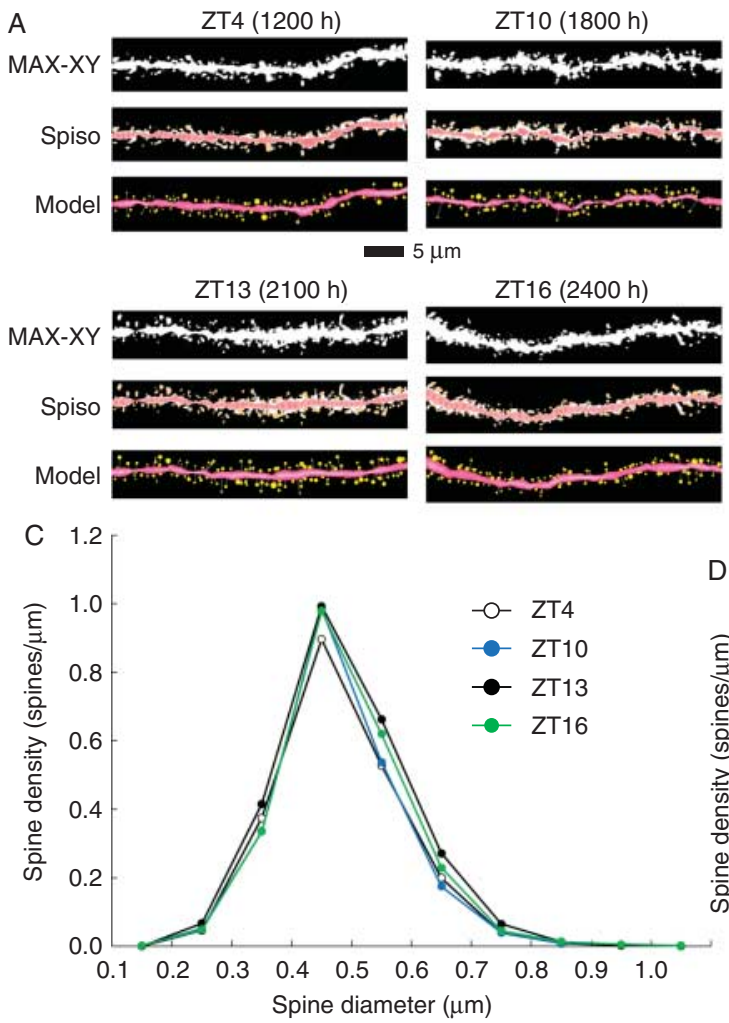

Figure 1

Diurnal change of the spine density in hippocampal CA1 pyramidal neurons. Spines were analyzed along the secondary dendrites of CA1 pyramidal neurons in the stratum radiatum every $3 \mathrm{~h}$. (A) Representative images of confocal micrographs; the spines along dendrite at Zeitgeber time 4 (ZT4, 1200 h), ZT10 (1800 h), ZT13 (2100 h), and ZT16 (2400 h). Maximal intensity projections onto $X Y$ plane from z-series confocal micrographs (MAX-XY), images analyzed by Spiso-3D (Spiso), and threedimensional model illustrations (model) are shown together. Bar, $5 \mu \mathrm{m}$. (B) Diurnal change of the total spine density in the hippocampus. Vertical axis represents the average number of spines per $1 \mu \mathrm{m}$ of dendrite. Data are represented as mean \pm s.E.M. (C) Histogram of spine head diameters at ZT4 (closed white circle), ZT10 (closed blue circle), ZT13 (closed black circle), by Tukey-Kramer post hoc multiple comparisons test. A difference was considered significant at a value of ${ }^{*} P<0.05$ or ${ }^{* *} P<0.01$.

\section{Results}

Spine density showed diurnal change in the in vivo fixed hippocampus

We investigated the diurnal change of dendritic spine density in the hippocampus. Lucifer Yellow-injected neurons in hippocampal slices from 12-week-old male rats were imaged using confocal laser scan microscopy (Fig. 1A). We analyzed secondary branches in the apical
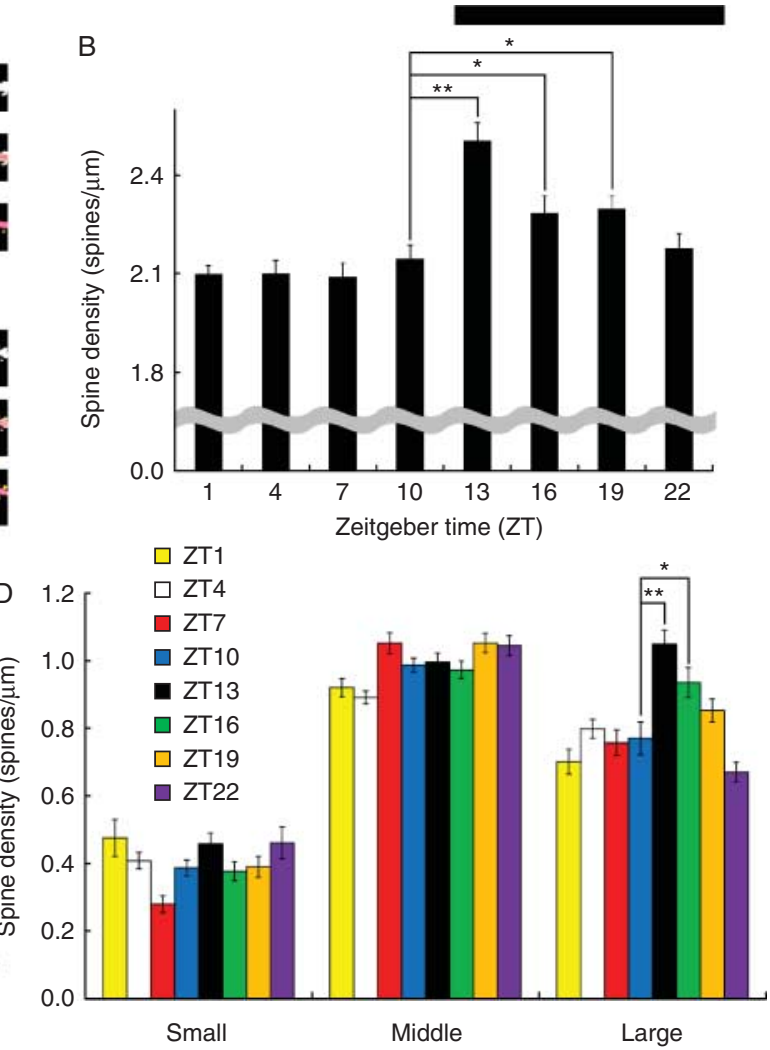

and ZT16 (closed green circle). (D) Density of three subtypes of spines at ZT1 (yellow column), ZT4 (white column), ZT7 (red column), ZT10 (blue column), ZT13 (black column), ZT16 (green column), ZT19 (orange column), and ZT22 (purple column). From left to right, small-head spines (small), middle-head spines (middle), and large-head spines (large) type. Data are represented as mean \pm s.E.M. The statistical significance was examined using one-way ANOVA followed by the Tukey-Kramer post hoc multiple comparisons test. The significance yielded: ${ }^{*} P<0.01,{ }^{*} P<0.05$ vs 'ZT10'. For each period of time, we investigated three to four rats, six to eight slices, 30-40 neurons, 60-80 dendrites, and $\sim 6000-8000$ spines. A full colour version of this figure is available at http://dx.doi.org/10.1530/JOE-15-0078.

Published by Bioscientifica Ltd. 
dendrites located $100-250 \mu \mathrm{m}$ away from the pyramidal cell body and in the middle of the stratum radiatum in the CA1 region.

Total spine density analysis The total spine density showed the time-dependent change as follows: 2.10

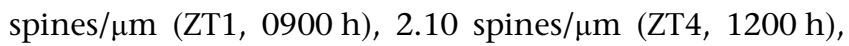

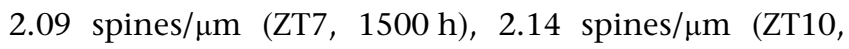

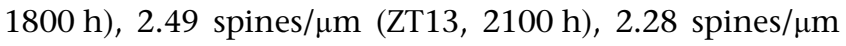

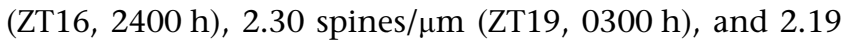

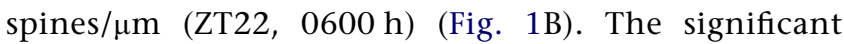
increase of the spine density was observed between ZT10 and ZT13. The spine density moderately decreased, from ZT13 to ZT19, but was still higher than at ZT10. The similar tendency was observed in its CORT level, higher in the awake state (ZT12-ZT22) and lower in the sleep state (ZT1-ZT10) (Higo et al. 2011; Supplementary Fig. S3, see section on supplementary data given at the end of this article).

Spine head diameter analysis The morphological changes in spine head diameter were assessed. We classified the spines into three categories using their head diameter: $0.2-0.4 \mu \mathrm{m}$ as small-head spines, $0.4-$ $0.5 \mu \mathrm{m}$ as middle-head spines, and larger than $0.5 \mu \mathrm{m}$ as large-head spines. Upon waking at ZT13, the density of large-head spines was considerably increased from 0.77 spines/ $\mu \mathrm{m}$ (at ZT10) to 1.05 spines/ $\mu \mathrm{m}$ (Fig. 1C and D). The spine density for small- and middle-head spines was not significantly altered.

\section{Depletion of circulating CORT suppressed the change of the spine density}

To clarify the effect of CORT on the observed diurnal change of the spine density, ADX was performed, depleting circulating CORT. In addition, metyrapone (a specific inhibitor of CORT synthesis by P450(11ß)) was administrated subcutaneously to eliminate the rise of CORT level upon waking.

Total spine density analysis The spine density of ADX rats was examined at ZT4 and ZT13. ADX decreased spine density at ZT13 from 2.49 to 2.09 spines $/ \mu \mathrm{m}$. ADX prevented the increase of spine density at ZT13, whereas there was no effect at ZT4 (Fig. 2A and B; Supplementary Fig. S4A, see section on supplementary data given at the end of this article). When $1 \mathrm{mg} / \mathrm{kg}$ body weight CORT was injected subcutaneously into ADX rats at ZT11, the spine density increased at ZT13, implying that the low spine density by ADX was rescued by CORT supplementation (Fig. 3A and B).

Metyrapone was administrated subcutaneously at ZT9, $4 \mathrm{~h}$ before the decapitation. The treatment of metyrapone prevented the increase of the spine density at ZT13 (2.12 spines/ $\mu \mathrm{m})$, resulting in the same level with the sleep state (Fig. 4A and B). Treatment of vehicle (sesame oil) showed no effect (Supplementary Fig. S4A). These results support that the increase of the spine density during the awake state is induced by the elevation of CORT released from the adrenal gland.

Spine head diameter analysis By ADX, the increase of the large-head spine density at ZT13 was suppressed, resulting in the same spine density for all the three subpopulations as those in intact rats at ZT4. In addition, ADX did not affect the spine density for all three subpopulations at ZT4 (Fig. 2C and D). CORT injection into ADX rats rescued the decrease of large-head spine density, resulting in the same spine density for all three subpopulations as those in intact rats at ZT13 (Fig. 3C and D).

The treatment of metyrapone prevented the increase of both the middle- and large-head spine density at ZT13. In the control experiment, sesame oil had no effect on all three subpopulations of the spine density (Fig. 4C and D), implying that the stress from the injection procedure had no effect.

Determination of CORT levels in the hippocampus after the injection of CORT The concentration of CORT in the ADX rats at $2 \mathrm{~h}$ after the CORT injection was determined by the MS analysis. Chromatographic profiles for the fragmented ions of CORT $(m / z=121)$ showed a single peak with its retention time of 2.83 min (Supplementary Fig. S5A and B, see section on supplementary data given at the end of this article). The average concentration of CORT in the hippocampus was $23.7 \mathrm{ng} / \mathrm{g}$ wet weight (68.4 nM; $n=3)$, slightly higher than that in the CSF of intact rats in the awake state $(\sim 30 \mathrm{nM})$. Upon CORT injection into ADX rats, the hippocampal CORT became much higher than that in ADX rats without CORT injection (6.9 nM; Higo et al. 2011).

Low level CORT rapidly increased spine density in isolated hippocampal slices

Since the spine density in the in vivo hippocampus changed within $3 \mathrm{~h}$, we investigated the molecular mechanism of this rapid spine change by using isolated hippocampal slices. Since the physiological level of CORT

Published by Bioscientifica Ltd 

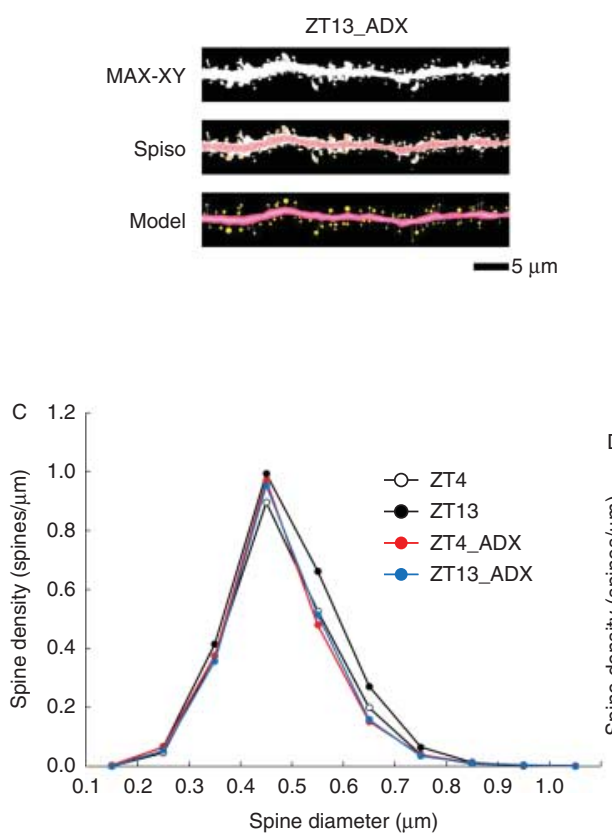

Figure 2

Effect of adrenalectomy (ADX) on the diurnal change of the spine density. Endogenous CORT was depleted by ADX, and spine density was analyzed at ZT4 (ZT4_ADX) and ZT13 (ZT13_ADX). (A) Representative images of confocal micrographs; the spines along the dendrite of ADX rats at $\mathrm{ZT} 13$. Maximal intensity projections onto $X Y$ plane from z-series confocal micrographs (MAX-XY), images analyzed by Spiso-3D (Spiso), and threedimensional model illustrations (model) are shown together. Bar, $5 \mu \mathrm{m}$. (B) Effect of ADX on the total spine density. Vertical axis represents the average number of spines per $1 \mu \mathrm{m}$ of dendrite. Data are represented as mean \pm S.E.M. (C) Histogram of spine head diameters at ZT4 (closed white

at ZT13 in CSF is $\sim 30 \mathrm{nM}$ (Higo et al. 2011), we investigated the effect by the application of $30 \mathrm{nM}$ CORT on the dendritic spine density in hippocampal acute slices.

Total spine density analysis Following a 1-h treatment with $30 \mathrm{nM}$ CORT on isolated hippocampal slices, treated dendrites had more spines (1.40 spines/ $\mu \mathrm{m})$ than control dendrites (1.16 spines $/ \mu \mathrm{m})$ (Fig. $5 \mathrm{~A}$ and $\mathrm{B}$ ). It should be noted that the CORT level in control slices was $\sim 2 \mathrm{nM}$ (Hojo et al. 2011, Ooishi et al. 2012). In the control condition, the spine density did not decline, even after $5 \mathrm{~h}$ (Supplementary Fig. S6, see section on supplementary data given at the end of this article), indicating the slices were sufficiently alive.

Blocking GR by $10 \mu \mathrm{M}$ RU486 completely abolished the CORT-induced spinogenesis (1.14 spines/ $\mu \mathrm{m}$; Fig. 6A and B; Supplementary Fig. S4B). Although GR is known as a nuclear translocation receptor, the inhibition of gene transcriptions by $4 \mu \mathrm{M}$ actinomycin $\mathrm{D}$ had no effect on the
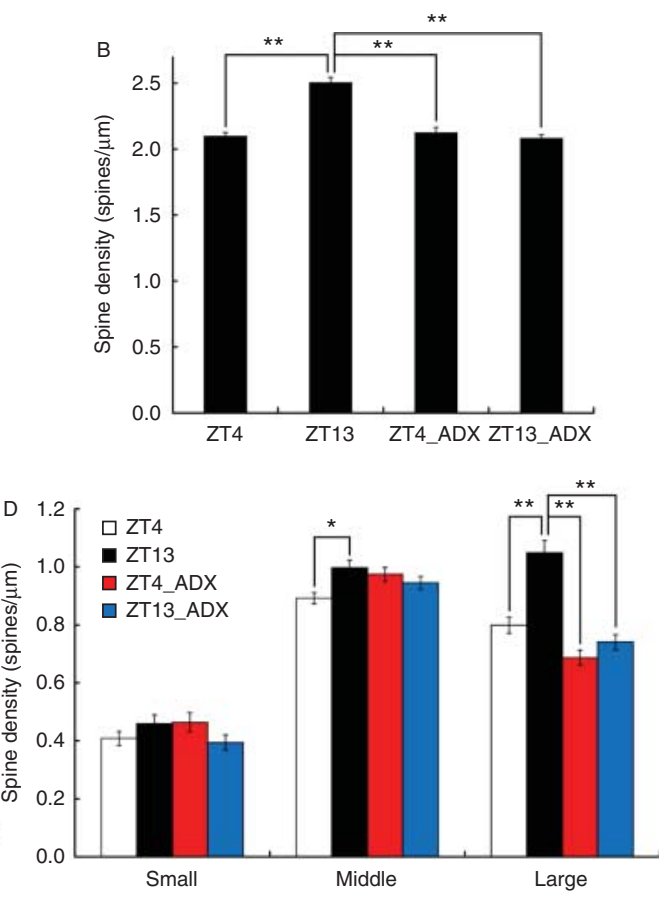

circle) and ZT13 (closed black circle) and of ADX rats at ZT4 (closed red circle) and ZT13 (closed blue circle). (D) Density of three subtypes of spines at ZT4 (white column) and ZT13 (black column) and in ADX rats at ZT4 (red column) and ZT13 (blue column). From left to right, small-head spines (small), middle-head spines (middle), and large-head spines (large) type. Data are represented as mean \pm s.E.M. The statistical significance yielded: $* * P<0.01$ and $* P<0.05$. For each condition, we investigated three to four rats, six to eight slices, 30-40 neurons, $60-80$ dendrites, and $\sim 6000-8000$ spines. A full colour version of this figure is available at http://dx.doi.org/ 10.1530/JOE-15-0078.

CORT-induced spinogenesis (1.40 spines/ $\mu \mathrm{m})$. CHX, an inhibitor of protein synthesis, completely abolished the CORT-induced spinogenesis (1.14 spines/ $\mu \mathrm{m})$ at $20 \mu \mathrm{M}$ (Fig. 6C and Supplementary Fig. S4B). These results suggest that the observed CORT-induced spinogenesis is elicited by synaptic GR (Ooishi et al. 2012), which can trigger signaling cascades rapidly ( $\sim 1 \mathrm{~h})$. It should be noted that these blockers and inhibitors alone did not significantly affect the spine density within experimental error (Supplementary Fig. S7A and B, see section on supplementary data given at the end of this article), indicating that the observed inhibitory effects are not simply due to the blocker's nonspecific suppressive effects. Blocking mineralocorticoid receptors (MR) by $10 \mu \mathrm{M}$ spironolactone did not affect the spine density (1.35 spines/ $\mu \mathrm{m})$.

Spine head diameter analysis Upon treatment with $30 \mathrm{nM}$ CORT in $1 \mathrm{~h}$, both the small- and middle-head spines increased significantly (Fig. 5C and D).

Published by Bioscientifica Ltd 

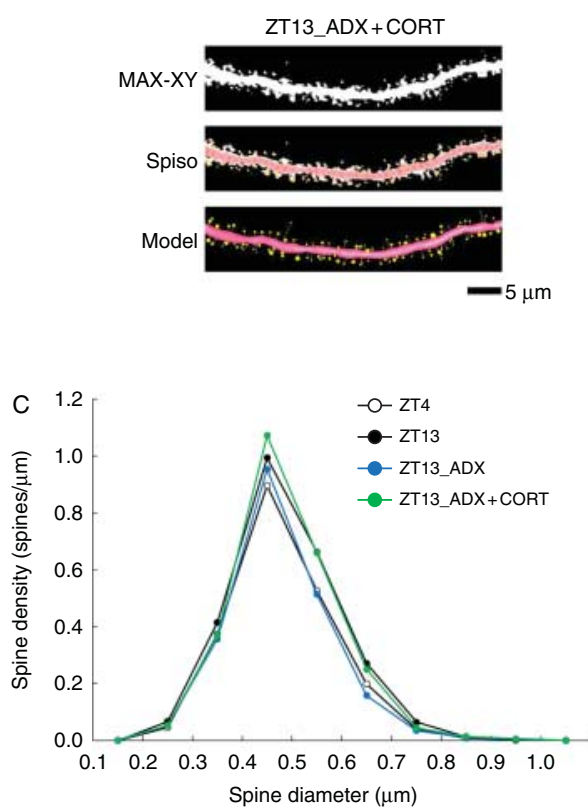

Figure 3

Effect of the administration of CORT on ADX rats. Exogenous CORT was administered subcutaneously ( $1 \mathrm{mg} / \mathrm{kg}$ body weight) on ADX rats at ZT11. Spine density was analyzed at ZT13 (ZT13_ADX+CORT). (A) Representative images of confocal micrographs; the spines along the dendrite of ADX rats with CORT administration. Maximal intensity projections onto XY plane from z-series confocal micrographs (MAX-XY), images analyzed by Spiso-3D (Spiso), and three-dimensional model illustrations (model) are shown together. Bar, $5 \mu \mathrm{m}$. (B) Effect of the administration of CORT on the total spine density. Vertical axis represents the average number of spines per $1 \mu \mathrm{m}$ of dendrite. Data are represented as mean \pm S.E.M. (C) Histogram of spine head diameters at ZT4 (closed white circle) and ZT13 (closed black

The density of large-head spines was not significantly altered. Blocking GR abolished the effect of CORT by decreasing the density of middle-head spines (Supplementary Fig. S8A, see section on supplementary data given at the end of this article).

\section{Inhibition of kinases suppressed CORT-induced spinogenesis}

To identify the kinases that are included within the downstream of GR signaling, the CORT-induced spinogenesis with co-treatments of specific inhibitors for kinases was investigated.

Total spine density analysis Application of $10 \mu \mathrm{M} \mathrm{H}-89$ (PKA inhibitor), $10 \mu \mathrm{M}$ chelerythrine (PKC inhibitor), $25 \mu \mathrm{M}$ U0126 (ERK inhibitor), and $10 \mu \mathrm{M}$ LIMK inhibitor prevented the effect of $30 \mathrm{nM}$ CORT (Fig. 7A and B; Supplementary Fig. S4B). On the other hand, the
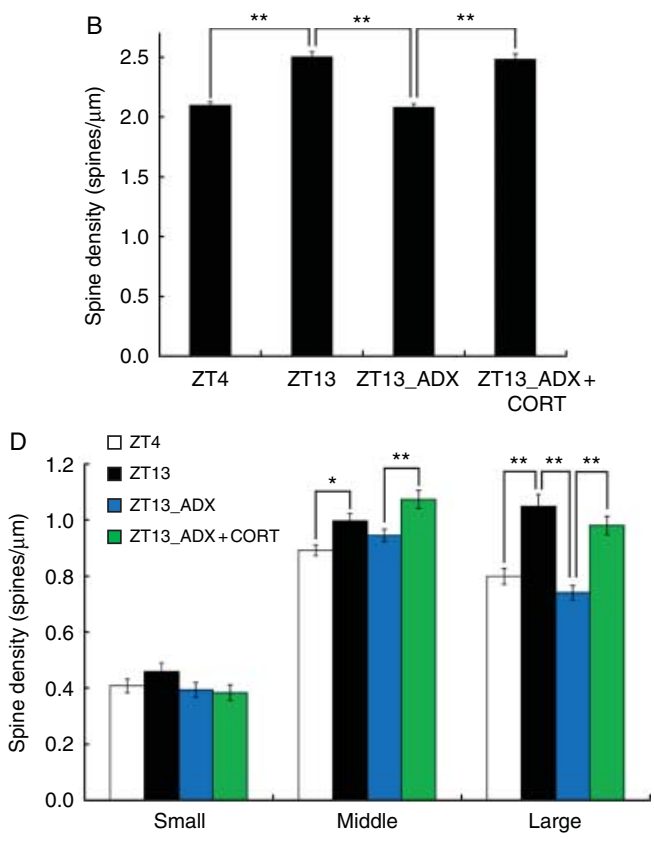

circle), in ADX rats at ZT13 (closed blue circle), and in CORT-administered ADX rats (closed green circle). (D) Density of three subtypes of spines at ZT4 (white column) and ZT13 (black column), in ADX rats at ZT13 (blue column), and in CORT-administered ADX rats (green column). From left to right, small-head spines (small), middle-head spines (middle), and large-head spines (large) type. Data are represented as mean \pm s.E.M. The statistical significance yielded: $* * P<0.01,{ }^{*} P<0.05$ vs 'ZT13' and 'ZT13_ADX'. For each condition, we investigated three to four rats, six to eight slices, 30-40 neurons, $60-80$ dendrites, and $\sim 6000-8000$ spines. A full colour version of this figure is available at http://dx.doi.org/10.1530/JOE-15-0078.

application of $10 \mu \mathrm{M}$ SP600125 (JNK inhibitor) did not alter the effect of CORT. These results indicate that $30 \mathrm{nM}$ CORT promoted the spinogenesis via PKA, PKC, ERK MAPK, and LIMK signaling pathways. Since the concentrations of inhibitors applied are at their recommended levels (Birnbaum et al. 2004, Venugopal et al. 2007, Hammond et al. 2008, Benakanakere et al. 2010, Scott et al. 2010), the observed inhibitory effects are not artifacts due to the excess amount of inhibitors. These kinase inhibitors alone did not significantly affect the spine density (Supplementary Fig. S7C).

Spine head diameter analysis Inhibiting PKA, ERK, and LIMK abolished the effect of CORT, decreasing the density of small-head spines (Fig. 7C and D). Inhibiting PKC decreased the density of middle-head spines. On the other hand, inhibiting JNK had no effect on each class of the spine density, implying that these effects of kinase inhibitors were not nonspecific.

Published by Bioscientifica Ltd. 
A
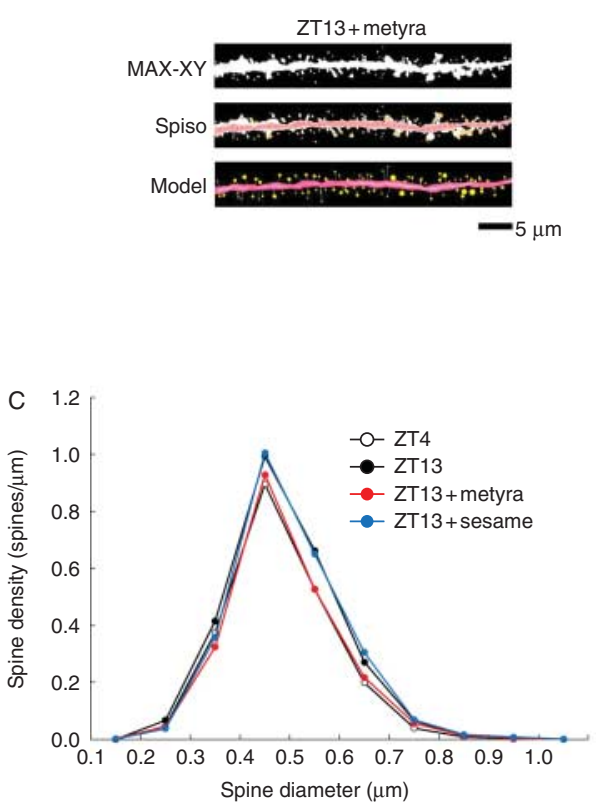

Figure 4

Effect from the inhibition of CORT synthesis on the diurnal change of the spine density. The synthesis of CORT at the adrenal gland was inhibited by metyrapone. Vehicle (sesame oil (ZT13 + sesame)) or $50 \mathrm{mg} / \mathrm{kg}$ body weight metyrapone was administered subcutaneously at ZT9 and spine density was analyzed at ZT13 (ZT13 + metyra). (A) Representative images of confocal micrographs; the spines along dendrite of metyrapone administered group. Maximal intensity projections onto XY plane from z-series confocal micrographs (MAX-XY), images analyzed by Spiso-3D (Spiso), and threedimensional model illustrations (model) are shown together. Bar, $5 \mu \mathrm{m}$. (B) Effect of metyrapone on the total spine density. Vertical axis represents the average number of spines per $1 \mu \mathrm{m}$ of dendrite. Data are represented as mean \pm s.E.M. (C) Histogram of spine head diameters at ZT4 (closed white

\section{Discussion}

The current study showed that the spine density of CA1 pyramidal neurons in the hippocampus changed along the circadian cycles of the CORT level. The moderately rapid increase of the spine density from the sleep state to the awake state is probably induced by the rise in CORT level. Such a moderately rapid spine increase might be induced by kinase signaling, including PKA PKC, ERK MAPK, and LIMK.

\section{Diurnal change of spine density in the hippocampus and cortex}

The observed increase in spine density $(\sim 20 \%)$ in the hippocampus could facilitate memory performance (Leuner \& Shors 2004, Luine \& Frankfurt 2012), and the spine increase may have a correlation with the diurnal change of the hippocampal-dependent memory performance (Ruby et al. 2008).
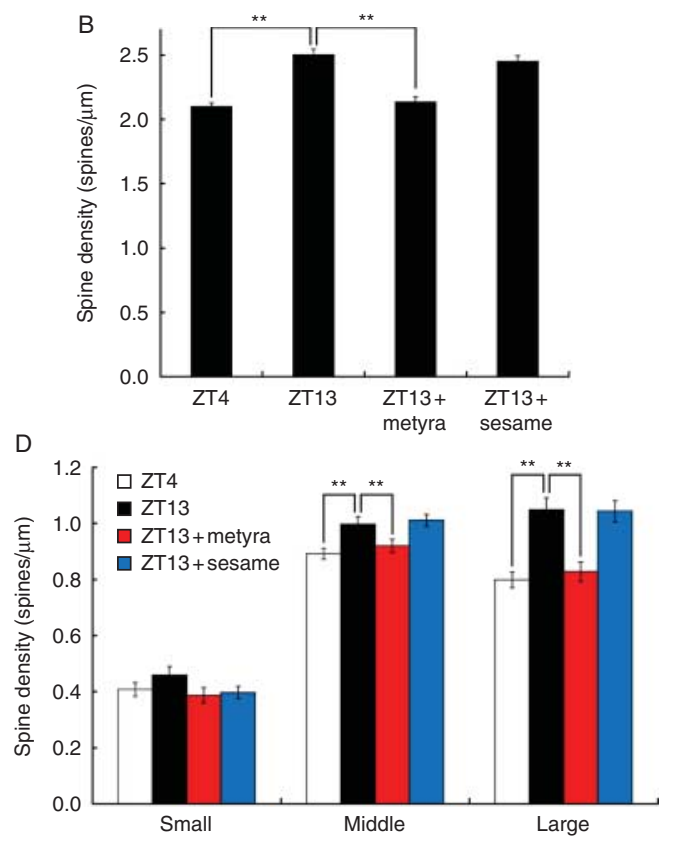

circle) and ZT13 (closed black circle), in metyrapone administered group (closed red circle), and in vehicle administered group (closed blue circle). (D) Density of three subtypes of spines at ZT4 (white column) and ZT13 (black column), in metyrapone administered group (red column), and in vehicle administered group (blue column). From left to right, small-head spines (small), middle-head spines (middle), and large-head spines (large) type. Data are represented as mean \pm s.E.M. The statistical significance yielded: ${ }^{*} P<0.01$ vs ' $Z$ T13'. For each condition, we investigated three to four rats, six to eight slices, 30-40 neurons, $60-80$ dendrites, and $\sim 6000-8000$ spines. A full colour version of this figure is available at http://dx.doi.org/10.1530/JOE-15-0078.

The change of the spine density along the sleep-wake cycle is also observed in the cortex, including infralimbic cortex layer III (Perez-Cruz et al. 2009), sensorimotor cortex layer V (Maret et al. 2011), barrel cortex layer V (Yang \& Gan 2012), and primary cerebral cortex layer II/III (Hayashi et al. 2013). In these studies, the spine density increases also in the awake state and decreases in the sleep state, probably resulting in the diurnal change of the cortex-dependent memory performance (Liston et al. 2013). However, the detailed mechanisms are not clarified.

By ADX and the s.c. administration of metyrapone, we demonstrated that the rise in CORT level upon waking increased spine density in the hippocampus. Perez-Cruz et al. (2009) show that the chronic restraint stress diminishes the wake-induced increase of the spine density in the cortex. These results imply that the increase of spines in the cortex might also be due to the change in

Published by Bioscientifica Ltd 
A
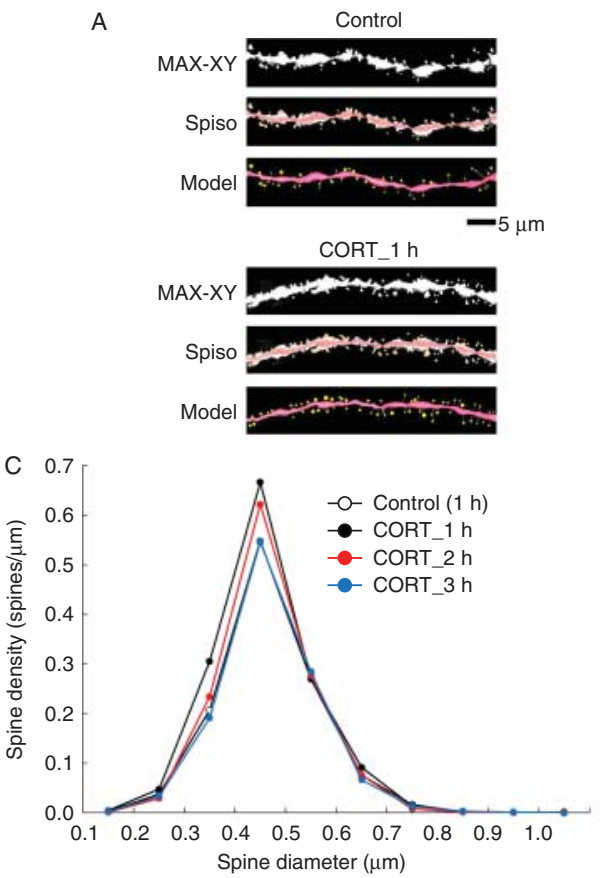

Figure 5

Time dependency of CORT effects on the spine density in isolated hippocampal slices. Spines in isolated hippocampal slices were analyzed along the secondary dendrites of CA1 pyramidal neurons in the stratum radiatum. $30 \mathrm{nM}$ CORT was treated on acute hippocampal slices for $1 \mathrm{~h}$ (CORT_1 h), 2 h (CORT_2 h), and 3 h (CORT_3 h) in ACSF. (A) Representative images of confocal micrographs; the spines along dendrite without drugs (control) and with $30 \mathrm{nM}$ of CORT treatment for $1 \mathrm{~h}$. Maximal intensity projections onto $X Y$ plane from z-series confocal micrographs (MAX-XY), images analyzed by Spiso-3D (Spiso), and three-dimensional model illustrations (model) are shown together. Bar, $5 \mu \mathrm{m}$. (B) Time dependency of CORT effects on the total spine density. As a control, no treatment with CORT for $1 \mathrm{~h}$ (control $(1 \mathrm{~h})$ ) is shown. Vertical axis represents the average number of spines per $1 \mu \mathrm{m}$ of dendrite. Data are represented as

CORT level, since the chronic stress is known to diminish the rhythm of the CORT level (Miyazaki et al. 2013).

\section{Contribution of slow effects of GR to spine changes may be weak}

GR is known as a nuclear translocation receptor, regulating gene transcriptions and protein syntheses. These processes may take $5-6 \mathrm{~h}$, which is slower than the moderately rapid spine increase that appeared within $3 \mathrm{~h}$ (from ZT10 to ZT13), for in vivo fixed hippocampus (Fig. 1). From DNA microarray analysis, the genes that were regulated by CORT in the hippocampus are investigated comprehensively (Morsink et al. 2006). mRNAs of typical synaptic proteins, including NMDA-R, AMPA-R, PSD95, synapsin I, and synaptophisin, were not changed within $5 \mathrm{~h}$, upon CORT treatment. These results suggest that the observed moderately rapid increase $(<3 \mathrm{~h})$ in spine
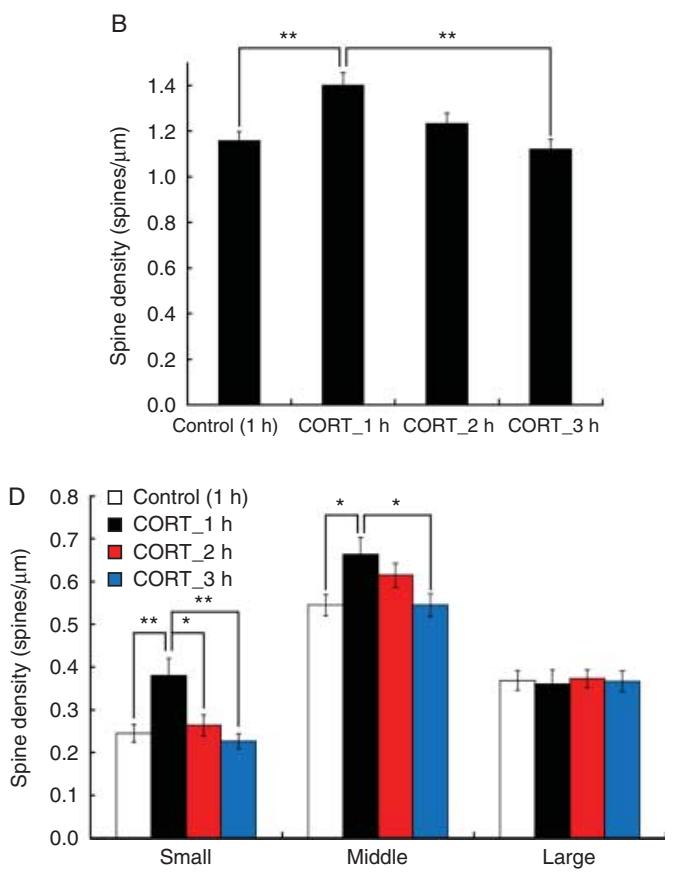

mean \pm S.E.M. (C) Histogram of spine head diameters after a $1 \mathrm{~h}$ treatment in ACSF without drugs (closed white circle) and with $30 \mathrm{nM}$ CORT for $1 \mathrm{~h}$ (closed black circle), $2 \mathrm{~h}$ (closed red circle), and $3 \mathrm{~h}$ (closed blue circle). (D) Density of three subtypes of spines after a $1 \mathrm{~h}$ treatment in ACSF without drugs (white column) and with $30 \mathrm{nM}$ CORT for $1 \mathrm{~h}$ (black column), $2 \mathrm{~h}$ (red column), and $3 \mathrm{~h}$ (blue column). From left to right, small-head spines (small), middle-head spines (middle), and large-head spines (large) type. Data are represented as mean \pm S.E.M. The statistical significance yielded: ${ }^{*} P<0.01$ and $* P<0.05$. For each condition, we investigated three to four rats, six to eight slices, 30-40 neurons, 60-80 dendrites, and $\sim 3000$ 4000 spines. A full colour version of this figure is available at http://dx.doi. org/10.1530/JOE-15-0078.

density upon waking may be too rapid to slow genomic effects of GR but might be driven by rapid kinase-driven effects as observed in isolated hippocampal slices.

\section{Rapid spine increase by physiologically low level CORT}

CORT-induced rapid spinogenesis via synaptic GR and kinases In isolated hippocampal slices, the application of $30 \mathrm{nM}$ CORT, approximately the same level with that in the CSF during the awake state (Higo et al. 2011), increased the spine density within $1 \mathrm{~h}$, from the control slices with a CORT level of $\sim 2 \mathrm{nM}$. The effect of CORT was blocked by RU486, implying that CORT effect was mediated by GR. Since RU486 suppresses not only GR but also progesterone receptor (PR), progesterone (PROG) effect should be considered. The treatment of slices with $10 \mathrm{nM}$ PROG (an approximately equal level in hippocampus (Hojo et al. 2009)) for $1 \mathrm{~h}$ did not significantly increase the spine

Published by Bioscientifica Ltd 
density within experimental error (data not shown), excluding the involvement of PROG and PR in the observed spinogenesis.

GR has been observed to localize within the postsynaptic structures with postembedding immunogold staining (Komatsuzaki et al. 2012, Ooishi et al. 2012), and there is increasing evidence that membrane-located GR could rapidly modulate synaptic functions by directly activating protein kinases pathways (Maggio \& Segal 2010, Groeneweg et al. 2012).

A
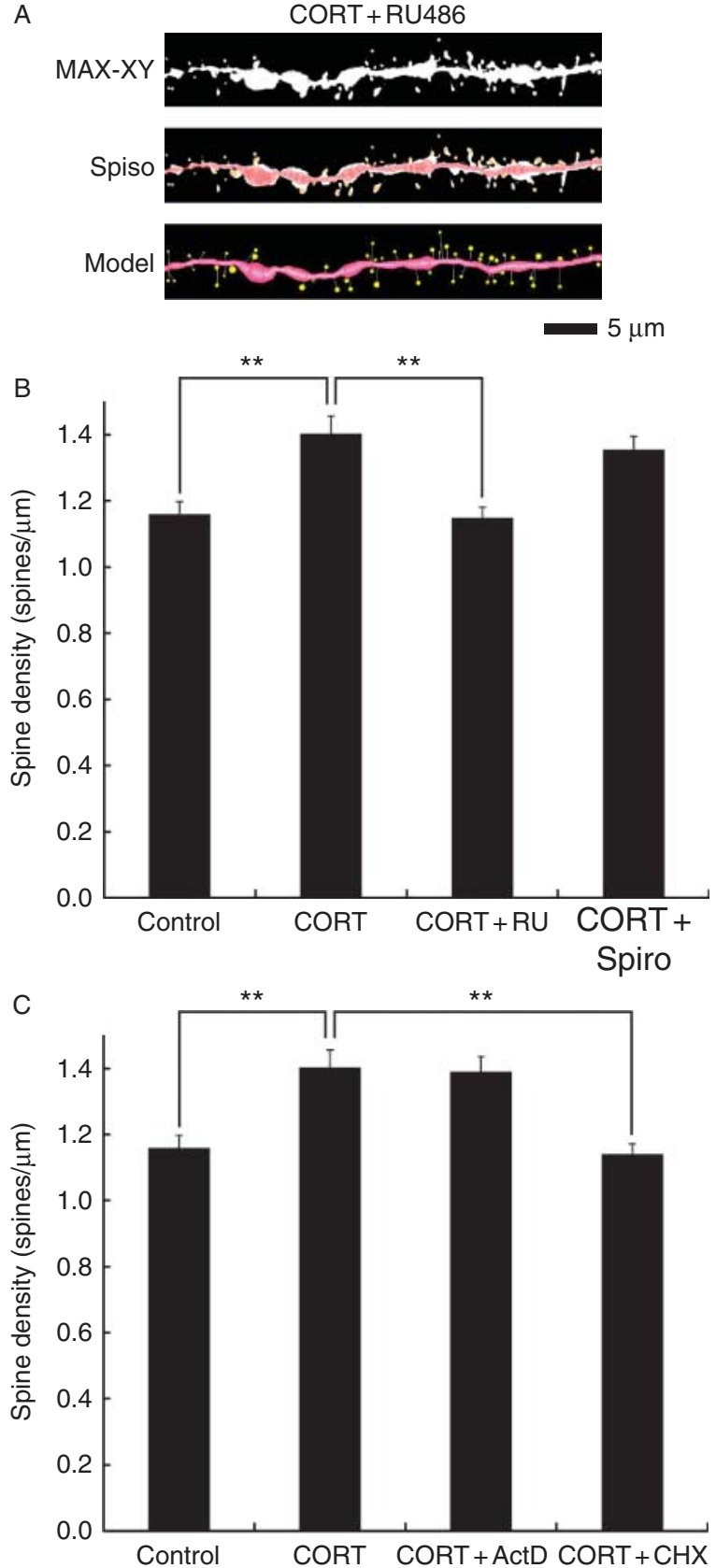

http://joe.endocrinology-journals.org DOI: 10.1530/JOE-15-0078
The current CORT-induced spinogenesis required PKA, PKC, ERK, and LIMK. Membrane GR-induced rapid PKA activation $(\sim 1 \mathrm{~h})$ has been demonstrated in rat the basolateral amygdala and prefrontal cortex (Roozendaal et al. 2002, Barsegyan et al. 2010), and the relationship between GR activity and PKC also been shown (Liu \& Chen 1995, ffrench-Mullen 1995). Both rapid ERK/MAPK activation $(\sim 15 \mathrm{~min})$ and LIMK activation ( $\sim 20 \mathrm{~min})$ through GR have been demonstrated in the hippocampal slices and cultures of hippocampal neurons (Jafari et al. 2012, Liston et al. 2013).

Downstream cascade of the kinase networks In hippocampal neurons, there may be a phosphorylation pathway: PKC $\rightarrow$ RhoA $\rightarrow$ ROCK $\rightarrow$ LIMK (Pilpel \& Segal 2004, Shi et al. 2009). LIMK phosphorylates cofilin, an actinassociated protein, resulting in assembly of actin cytoskeletal matrices (Yang et al. 1998, Bernstein \& Bamburg 2010, Yuen et al. 2011). GR modulation of spine morphology via LIMK and the cofilin dependent pathway is shown in primary cultured hippocampal neurons (Liston et al. 2013).

In the CA1 region, ERK cascade is known to couple with PKA and PKC via PKA $\rightarrow$ B-Raf $\rightarrow$ ERK, PKC $\rightarrow$ Raf1 $\rightarrow$ ERK in synaptic modulation (Roberson et al. 1999). One of the targets of ERK in spine reorganization might be cortactin, since ERK is known to phosphorylate cortactin, an actin-associated protein (Martinez-Quiles et al. 2004). Phosphorylated cortactin promotes actin fiber remodeling working with actin-related protein $2 / 3$ complex

\section{Figure 6}

Effects by the blockade of receptors and inhibition of gene expressions on CORT-induced spinogenesis. (A) Representative images of confocal micrographs; the spines along dendrite with $30 \mathrm{nM}$ CORT and $10 \mu \mathrm{M}$ RU486 (blocker of glucocorticoid receptor (GR)) (CORT+RU) for $1 \mathrm{~h}$. Maximal intensity projections onto $X Y$ plane from z-series confocal micrographs (MAX-XY), images analyzed by Spiso-3D (Spiso) and three-dimensional model illustrations (model) are shown together. Bar, $5 \mu \mathrm{m}$. (B) Effects from the blocker of GR and mineralocorticoid receptor (MR) on the CORTinduced spinogenesis. A $1 \mathrm{~h}$ treatment in ACSF without drugs (control), with $30 \mathrm{nM}$ CORT, with $30 \mathrm{nM}$ CORT and $10 \mu \mathrm{M}$ RU486 (CORT + RU), and with $30 \mathrm{nM}$ CORT and $10 \mu \mathrm{M}$ spironolactone (blocker of MR) (CORT+Spiro). Vertical axis represents the average number of spines per $1 \mu \mathrm{m}$ of dendrite. Data are represented as mean \pm s.E.M. (C) Effects by the inhibition in mRNA and protein synthesis on the CORT-induced spinogenesis. A $1 \mathrm{~h}$ treatment in ACSF without drugs (control), with $30 \mathrm{nM}$ CORT, with $30 \mathrm{nM}$ CORT and $4 \mu \mathrm{M}$ actinomycin $\mathrm{D}$ (translation inhibitor) (CORT+ActD), and with $30 \mathrm{nM}$ CORT and $20 \mu \mathrm{M}$ cycloheximide (transcription inhibitor) (CORT $+\mathrm{CHX}$ ). Vertical axis represents the average number of spines per $1 \mu \mathrm{m}$ of dendrite. Data are represented as mean \pm S.E.M. The statistical significance yielded: $* * P<0.01$ vs 'CORT'. For each drug treatment, we investigated three to four rats, six to eight slices, 30-40 neurons, 60-80 dendrites, and $\sim 3000-4000$ spines. A full colour version of this figure is available at http://dx.doi.org/10.1530/JOE-15-0078.

Published by Bioscientifica Ltd. 


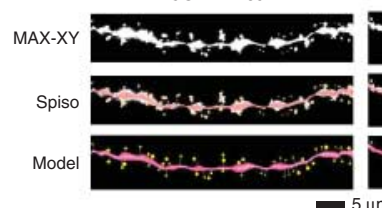

CORT + U0126
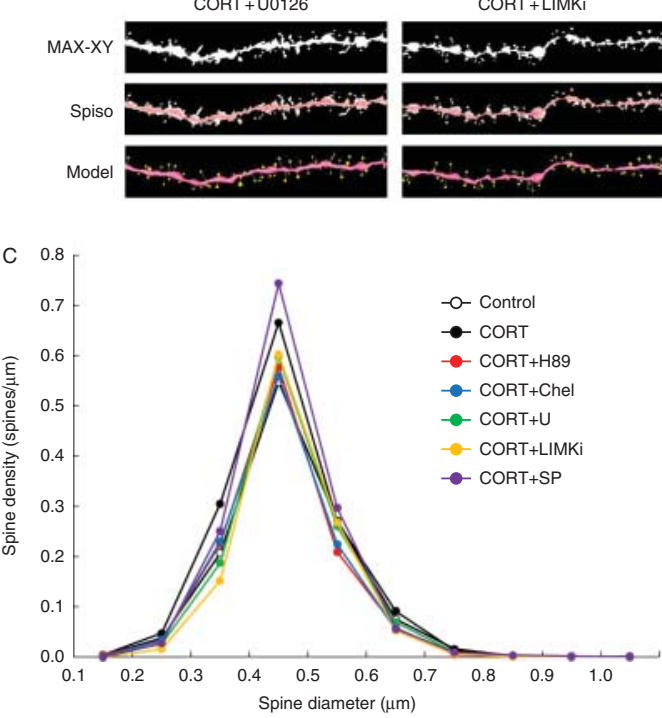

Figure 7

Suppression effects by kinase inhibitors on CORT-induced spinogenesis. Kinase inhibitors were co-treated with $30 \mathrm{nM}$ CORT on isolated hippocampal slices. A $1 \mathrm{~h}$ treatment in ACSF without drugs (control), with $30 \mathrm{nM}$ CORT (CORT), with $30 \mathrm{nM}$ CORT and $10 \mu \mathrm{M}$ H89 (PKA inhibitor)

(CORT+ H89), with $30 \mathrm{nM}$ CORT and $10 \mu \mathrm{M}$ chelerythrine (PKC inhibitor) (CORT+Chel), with $30 \mathrm{nM}$ CORT and $25 \mu \mathrm{M}$ U0126 (ERK inhibitor) $($ CORT $+U)$, with $30 \mathrm{nM}$ CORT and $10 \mu \mathrm{M}$ LIMK inhibitor (CORT+LIMKi), and with $30 \mathrm{nM}$ CORT and $10 \mu \mathrm{M}$ SP600125 (JNK inhibitor) (CORT+SP). (A) Representative images of confocal micrographs; the spines along dendrite with CORT and H89, with CORT and chelerythrine, with CORT and U0126, with CORT and LIMK inhibitor. Maximal intensity projections onto $X Y$ plane from z-series confocal micrographs (MAX-XY), images analyzed by Spiso-3D (Spiso), and three-dimensional model illustrations (model) are shown together. Bar, $5 \mu \mathrm{m}$. (B) Effects by the co-treatment of kinase inhibitors on the total spine density. Vertical axis represents the average number of spines per $1 \mu \mathrm{m}$ of dendrite. Data are represented as

(Weaver et al. 2001), as well as scaffold protein Shank in the PSD at the SH3 domain (Daly 2004).

Taken together, we can propose the schematic illustration of the CORT signaling pathway as shown in Fig. 8. Through this pathway, the rise in the CORT level upon waking may increase the spine density in the hippocampus.

\section{Comparison between in vivo hippocampus and in isolated hippocampal slices}

Diurnal change of CORT level in the in vivo hippocampus Circulating CORT level changes along the circadian
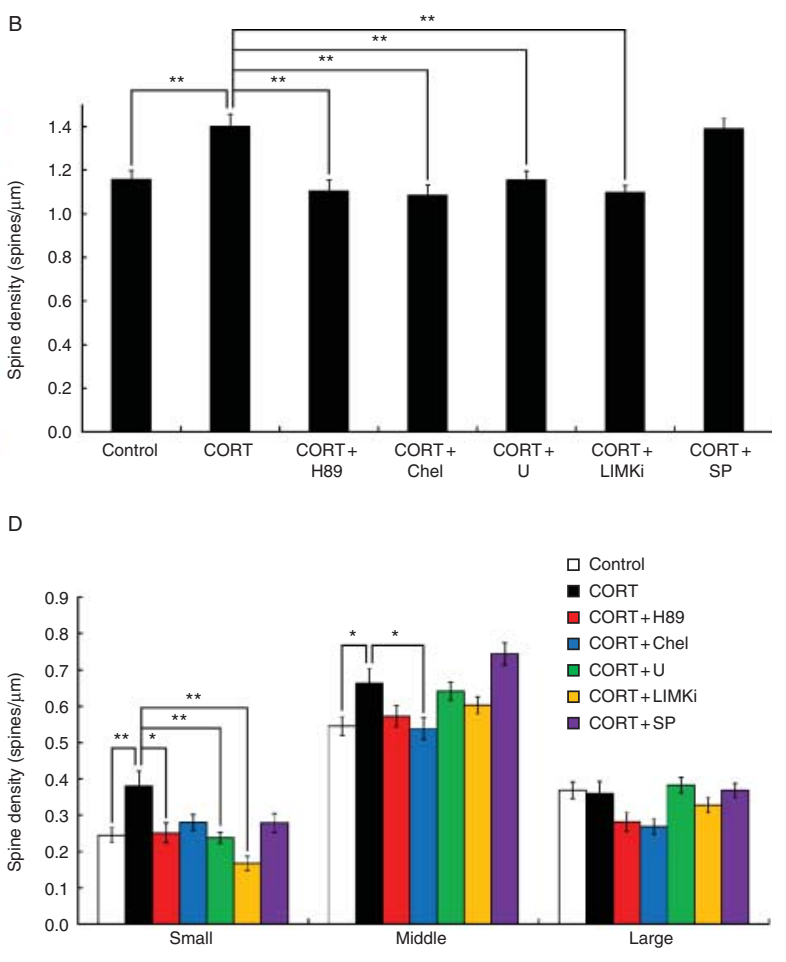

mean \pm S.E.M. (C) Histogram of spine head diameters after a $1 \mathrm{~h}$ treatment in ACSF without drugs (closed white circle), with CORT (closed black circle), with CORT and H89 (closed red circle), with CORT and chelerythrine (closed blue circle), with CORT and U0126 (closed green circle), with CORT and LIMK inhibitor (closed orange circle), and with CORT and SP600125 (closed purple circle). (D) Density of three subtypes of spines after a $1 \mathrm{~h}$ treatment in ACSF without drugs (white column), with CORT (black column), with CORT and H89 (red column), with CORT and chelerythrine (blue column), with CORT and U0126 (green column), with CORT and LIMK inhibitor (orange column), and with CORT and SP600125 (purple column). From left to right, small-head spines (small), middle-head spines (middle), and large-head spines (large) type. Data are represented as mean \pm s.E.M. The statistical significance yielded: $* * P<0.01, * P<0.05$ vs 'CORT'. For each drug treatment, we investigated three to four rats, six to eight slices, 30-40 neurons, $60-80$ dendrites, and $\sim 3000-4000$ spines. A full colour version of this figure is available at http://dx.doi.org/10.1530/JOE-15-0078.

rhythm (Migeon et al. 1956, Moore \& Eichler 1972). The diurnal change of the CORT level in the hippocampus has also been shown by using the microdialysis in combination with RIA (Linthorst \& Reul 2008, Qian et al. 2012). These studies, however, did not show the absolute CORT concentration in the hippocampus. On the other hand, our previous study showed the diurnal change of the CORT level in the CSF: $\sim 30 \mathrm{nM}$ in the awake state and $\sim 3 \mathrm{nM}$ in the sleep state (Higo et al. 2011; Supplementary Fig. S3). The concentration was determined with LC-MS/MS quantitatively. Therefore, we applied $30 \mathrm{nM}$ CORT to investigate the effect from the rise in CORT upon waking.

Published by Bioscientifica Ltd 


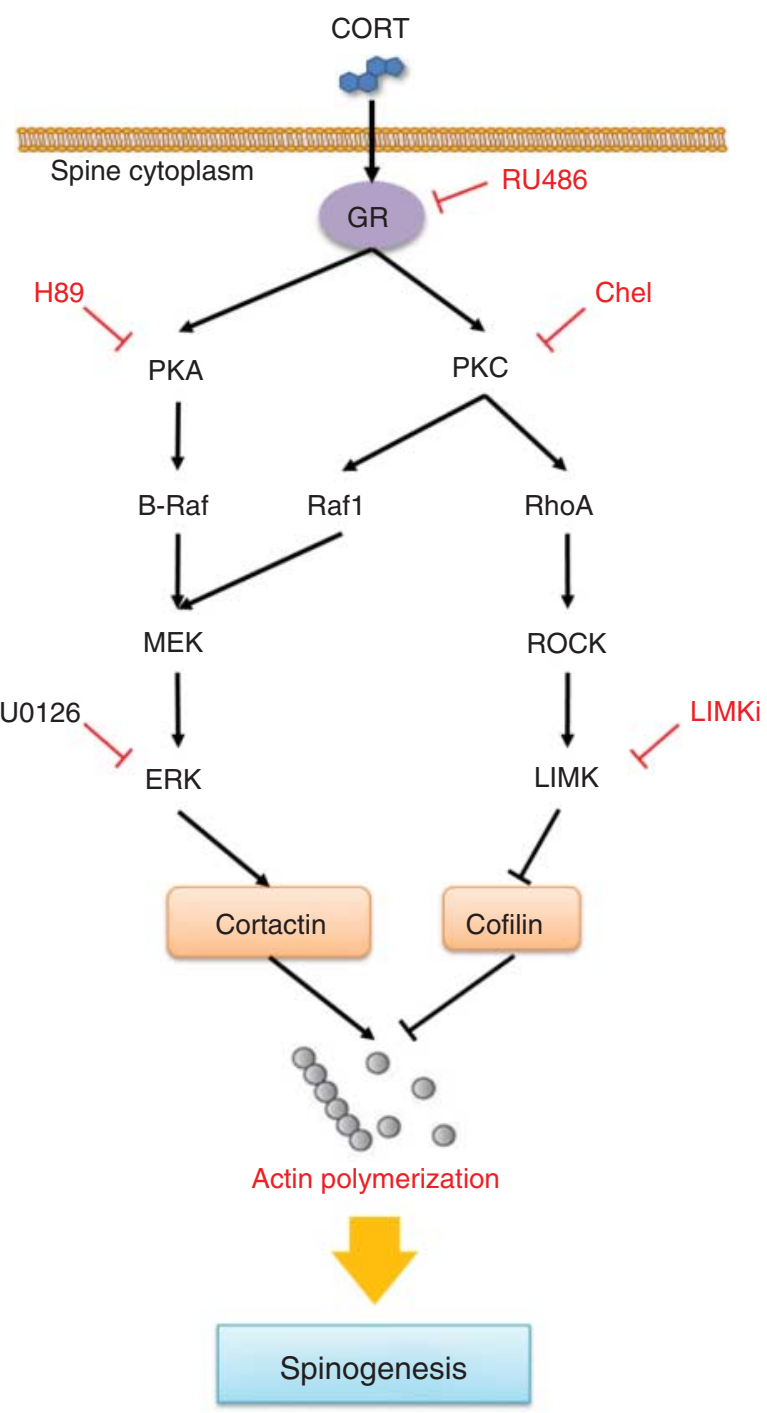

Figure 8

Schematic illustration of CORT-driven multiple kinase pathways. Upon binding of CORT, GR induces the sequential activation of PKA, PKC, ERK MAPK, and LIMK. LIMK phosphorylates cofilin, resulting in actin reorganization and subsequent spinogenesis, whereas ERK/MAPK phosphorylates cortactin. A full colour version of this figure is available at http://dx.doi.org/10.1530/JOE-15-0078.

Two hours after the s.c. injection of $1 \mathrm{mg} / \mathrm{kg}$ body weight CORT on ADX rats, the level of CORT increased to $\sim 70 \mathrm{nM}$, which induced the increase of the spine density, especially large-head spine density. It was also demonstrated that after the s.c. injection of $1 \mathrm{mg} / \mathrm{kg}$ body weight CORT, the CORT level was elevated for 15-120 min in the hippocampus (Droste et al. 2008).

Conditions in isolated hippocampal slices The isolated hippocampal slices, used for the spine analysis, have very low CORT because CORT was leaked to the ACSF during the incubation. The CORT level was $\sim 2 \mathrm{nM}$, which was determined by the MS analysis (Hojo et al. 2011, Komatsuzaki et al. 2012, Ooishi et al. 2012). Following an exposure to anesthesia and the sacrifice of rats, a high-dose of CORT $(\sim 1 \mu \mathrm{M})$ is secreted from the adrenal cortex and readily reaches the brain. Therefore, the CORT concentration in the freshly isolated hippocampus was 150 $500 \mathrm{nM}$ (Komatsuzaki et al. 2012). Incubation for $2 \mathrm{~h}$ is enough to wash out such a high level of CORT from hippocampal slices (Supplementary Fig. S1). For these reasons, the current spinogenesis occurred upon the increase in the CORT level from $\sim 2 \mathrm{nM}$ (control) to $30 \mathrm{nM}$. We have found that even $10 \mathrm{nM}$ CORT is enough to increase the spine density (Supplementary Fig. S9, see section on supplementary data given at the end of this article; Higo et al. 2011).

The spine density of isolated hippocampal slices (Figs 5, 6 and 7) is significantly lower than the spine density of in vivo fixed hippocampus (Figs 1, 2, 3 and 4). This may be due to the slicing with ice-cold water. Spines are known to disappear transiently when the cooled hippocampus is sliced (Kirov et al. 2004). Through recovery incubation of the slices for $2 \mathrm{~h}$ at room temperature, the spine density may increase to some extent. Perfusion fixation of the hippocampus in vivo was performed at body temperature $\left(\sim 37^{\circ} \mathrm{C}\right)$, much warmer than the fixation temperature of isolated hippocampal slices, preserving more spines.

\section{Concentration-dependent functions of non-stress level of CORT in the hippocampus}

The rapid functions of low level CORT have been reported in several studies. In hippocampal CA1 pyramidal neurons, $100 \mathrm{nM}$ of CORT facilitates the long-term potentiation induced by high-frequency stimulation (Wiegert et al. 2006), and 10-100 $\mathrm{nM}$ of CORT rapidly enhances the mEPSC frequency concentration dependently (Karst et al. 2005). This enhancement was via MR, whereas $30 \mathrm{nM}$ CORT-induced spinogenesis in the current study was not via MR but via GR. Since the affinity of GR for CORT is sufficiently high (dissociation constant $K_{\mathrm{d}}, 2.5-5 \mathrm{nM}$; Reul \& de Kloet 1985), GR is activated significantly by 30 nM CORT.

An inverted U-shape effect of CORT, including the amplitude of the population spike, is known to be weakened with the increase of CORT concentration above a certain threshold $(\sim 50 \mathrm{nM})$, whereas this effect is enhanced with the increase of CORT below the threshold (Diamond et al. 1992). Our earlier and current

Published by Bioscientifica Ltd. 
study showed that the CORT effect on spine density also had a U-shape relationship; 200-1000 nM CORT increased the spine density, $100 \mathrm{nM}$ CORT had no effect (threshold; Komatsuzaki et al. 2012), and 10-30 nM CORT increased the spine density (Higo et al. 2011, current study).

\section{Physiological importance of the circadian rhythms of spine and CORT}

Several studies have shown that memory performance depends on circadian rhythm (Eckel-Mahan \& Storm 2009). The score of the novel recognition task, the hippocampal-dependent memory test, shows diurnal change, higher in the awake state than in the sleep state (Ruby et al. 2008). In this study, the rhythm for memory performance is abolished by the disruption of the circadian system, most likely resulting in the disruption of CORT rhythm. The score of the rotarod motor learning task, which is dependent on motor cortex, is higher when training occurs during the circadian CORT peak than during the trough (Liston et al. 2013). The disruption of the CORT rhythm by the administration of CORT diminished the newly formed spines following motor training, resulting in impaired memory performance. This indicates that the rhythm of CORT not only increases spines but also supports the survival of newly formed spines. Other studies showed that the spatial memory (which is hippocampal dependent) and working memory (which is prefrontal cortex dependent) were impaired by ADX (Conrad et al. 1997, Mizoguchi et al. 2004). Furthermore, ADX rats show depression-like behavior in the rotarod test (Mizoguchi et al. 2008). Such memory and cognitive impairments are rescued by the regeneration of CORT rhythm by the combination of implant of the CORT pellet and oral CORT treatment, only during the awake phase. Even in humans, the retrieval of emotional and neutral texts is impaired by the suppression of the morning rise in cortisol (Rimmele et al. 2010). The current results are useful to explain the mechanisms of these diurnal changes of memory performance.

\section{Supplementary data}

This is linked to the online version of the paper at http://dx.doi.org/10.1530/ JOE-15-0078.

\section{Declaration of interest}

The authors declare that there is no conflict of interest that could be perceived as prejudicing the impartiality of the research reported.

\section{Funding}

This research was supported by JSPS Grants-in-Aid for Scientific Research.

\section{References}

Barsegyan A, Mackenzie SM, Kurose BD, McGaugh JL \& Roozendaal B 2010 Glucocorticoids in the prefrontal cortex enhance memory consolidation and impair working memory by a common neural mechanism. PNAS 107 16655-16660. (doi:10.1073/pnas.1011975107)

Benakanakere MR, Zhao J, Galicia JC, Martin M \& Kinane DF 2010 Sphingosine kinase- 1 is required for toll mediated $\beta$-defensin 2 induction in human oral keratinocytes. PLoS ONE 5 e11512. (doi:10.1371/journal.pone.0011512)

Bernstein BW \& Bamburg JR 2010 ADF/cofilin: a functional node in cell biology. Trends in Cell Biology 20 187-195. (doi:10.1016/j.tcb.2010. 01.001)

Birnbaum SG, Yuan PX, Wang M, Vijayraghavan S, Bloom AK, Davis DJ, Gobeske KT, Sweatt JD, Manji HK \& Arnsten AF 2004 Protein kinase C overactivity impairs prefrontal cortical regulation of working memory. Science 306 882-884. (doi:10.1126/science.1100021)

Buijs RM, Markman M, Nunes-Cardoso B, Hou YX \& Shinn S 1993 Projections of the suprachiasmatic nucleus to stress-related areas in the rat hypothalamus: a light and electron microscopic study. Journal of Comparative Neurology 335 42-54. (doi:10.1002/cne.903350104)

Chao HM, Choo PH \& McEwen BS 1989 Glucocorticoid and mineralocorticoid receptor mRNA expression in rat brain. Neuroendocrinology 50 365-371. (doi:10.1159/000125250)

Conrad CD, Lupien SJ, Thanasoulis LC \& McEwen BS 1997 The effects of type I and type II corticosteroid receptor agonists on exploratory behavior and spatial memory in the Y-maze. Brain Research 759 76-83. (doi:10.1016/S0006-8993(97)00236-9)

Daly RJ 2004 Cortactin signalling and dynamic actin networks. Biochemical Journal 382 13-25. (doi:10.1042/BJ20040737)

Diamond DM, Bennett MC, Fleshner M \& Rose GM 1992 Inverted-U relationship between the level of peripheral corticosterone and the magnitude of hippocampal primed burst potentiation. Hippocampus 2 421-430. (doi:10.1002/hipo.450020409)

Droste SK, de Groote L, Atkinson HC, Lightman SL, Reul JM \& Linthorst AC 2008 Corticosterone levels in the brain show a distinct ultradian rhythm but a delayed response to forced swim stress. Endocrinology 149 3244-3253. (doi:10.1210/en.2008-0103)

Eckel-Mahan KL \& Storm DR 2009 Circadian rhythms and memory: not so simple as cogs and gears. EMBO Reports 10 584-591. (doi:10.1038/ embor.2009.123)

Groeneweg FL, Karst H, de Kloet ER \& Joëls M 2012 Mineralocorticoid and glucocorticoid receptors at the neuronal membrane, regulators of nongenomic corticosteroid signalling. Molecular and Cellular Endocrinology 350 299-309. (doi:10.1016/j.mce.2011.06.020)

Hammond RS, Lin L, Sidorov MS, Wikenheiser AM \& Hoffman DA 2008 Protein kinase A mediates activity-dependent Kv4.2 channel trafficking. Journal of Neuroscience $\mathbf{2 8}$ 7513-7519. (doi:10.1523/JNEUROSCI. 1951-08.2008)

Hanani M 2012 Lucifer yellow - an angel rather than the devil. Journal of Cellular and Molecular Medicine 16 22-31. (doi:10.1111/j.1582-4934. 2011.01378.x)

Hayashi Y, Koyanagi S, Kusunose N, Okada R, Wu Z, Tozaki-Saitoh H, Ukai K, Kohsaka S, Inoue K, Ohdo S et al. 2013 The intrinsic microglial molecular clock controls synaptic strength via the circadian expression of cathepsin S. Scientific Reports 3 2744. (doi:10.1038/srep02744)

Higo S, Hojo Y, Ishii H, Komatsuzaki Y, Ooishi Y, Murakami G, Mukai H, Yamazaki T, Nakahara D, Barron A et al. 2011 Endogenous synthesis of corticosteroids in the hippocampus. PLoS ONE 6 e21631. (doi:10.1371/ journal.pone.0021631)

Published by Bioscientifica Ltd. 
Hojo Y, Higo S, Ishii H, Ooishi Y, Mukai H, Murakami G, Kominami T, Kimoto T, Honma S, Poirier D et al. 2009 Comparison between hippocampus-synthesized and circulation-derived sex steroids in the hippocampus. Endocrinology 150 5106-5112. (doi:10.1210/en. 2009-0305)

Hojo Y, Higo S, Kawato S, Hatanaka Y, Ooishi Y, Murakami G, Ishii H, Komatsuzaki Y, Ogiue-Ikeda M, Mukai H et al. 2011 Hippocampal synthesis of sex steroids and corticosteroids: essential for modulation of synaptic plasticity. Frontiers in Endocrinology 2 43. (doi:10.3389/fendo. 2011.00043)

Huang T-Y, Cherkas PS, Rosenthal DW \& Hanani M 2005 Dye coupling among satellite glial cells in mammalian dorsal root ganglia. Brain Research 1036 42-49. (doi:10.1016/j.brainres.2004.12.021)

Jacobson L \& Sapolsky R 1991 The role of the hippocampus in feedback regulation of the hypothalamic-pituitary-adrenocortical axis. Endocrine Reviews 12 118-134. (doi:10.1210/edrv-12-2-118)

Jafari M, Seese RR, Babayan AH, Gall CM \& Lauterborn JC 2012 Glucocorticoid receptors are localized to dendritic spines and influence local actin signaling. Molecular Neurobiology 46 304-315. (doi:10.1007/ s12035-012-8288-3)

Karst H, Berger S, Turiault M, Tronche F, Schütz G \& Joëls M 2005 Mineralocorticoid receptors are indispensable for nongenomic modulation of hippocampal glutamate transmission by corticosterone. PNAS 102 19204-19207. (doi:10.1073/pnas.0507572102)

Kim JJ, Song EY \& Kosten TA 2006 Stress effects in the hippocampus: synaptic plasticity and memory. Stress 9 1-11. (doi:10.1073/pnas. 0507572102)

Kirov SA, Petrak LJ, Fiala JC \& Harris KM 2004 Dendritic spines disappear with chilling but proliferate excessively upon rewarming of mature hippocampus. Neuroscience 127 69-80. (doi:10.1016/j.neuroscience. 2004.04.053)

Komatsuzaki Y, Hatanaka Y, Murakami G, Mukai H, Hojo Y, Saito M, Kimoto T \& Kawato $S 2012$ Corticosterone induces rapid spinogenesis via synaptic glucocorticoid receptors and kinase networks in hippocampus. PLOS ONE 7 e34124. (doi:10.1371/journal.pone.0034124)

Krugers HJ, Douma BR, Andringa G, Bohus B, Korf J \& Luiten PG 1997 Exposure to chronic psychosocial stress and corticosterone in the rat: effects on spatial discrimination learning and hippocampal protein kinase $\mathrm{C} \gamma$ immunoreactivity. Hippocampus 7 427-436. (doi:10.1002/ (SICI) 1098-1063(1997)7:4 < 427::AID-HIPO8 > 3.0.CO;2-F)

Leuner B \& Shors TJ 2004 New spines, new memories. Molecular Neurobiology 29 117-130. (doi:10.1385/MN:29:2:117)

Linthorst AC \& Reul JM 2008 Stress and the brain: solving the puzzle using microdialysis. Pharmacology, Biochemistry, and Behavior 90 163-173. (doi:10.1016/j.pbb.2007.09.019)

Liston C, Cichon JM, Jeanneteau F, Jia Z, Chao MV \& Gan W-B 2013 Circadian glucocorticoid oscillations promote learning-dependent synapse formation and maintenance. Nature Neuroscience 16 698-705. (doi:10.1038/nn.3387)

Liu X \& Chen YZ 1995 Membrane-mediated inhibition of corticosterone on the release of arginine vasopressin from rat hypothalamic slices. Brain Research 704 19-22. (doi:10.1016/0006-8993(95)01068-8)

Luine VN \& Frankfurt M 2012 Estrogens facilitate memory processing through membrane mediated mechanisms and alterations in spine density. Frontiers in Neuroendocrinology 33 388-402. (doi:10.1016/j. yfrne.2012.07.004)

Maggio N \& Segal M 2010 Corticosteroid regulation of synaptic plasticity in the hippocampus. ScientificWorldJournal 10 462-469. (doi:10.1100/tsw. 2010.48)

Maret S, Faraguna U, Nelson AB, Cirelli C \& Tononi G 2011 Sleep and waking modulate spine turnover in the adolescent mouse cortex. Nature Neuroscience 14 1418-1420. (doi:10.1038/nn.2934)

Martinez-Quiles N, Ho HY, Kirschner MW, Ramesh N \& Geha RS 2004 Erk/Src phosphorylation of cortactin acts as a switch on-switch off mechanism that controls its ability to activate N-WASP. Molecular and
Cellular Biology 24 5269-5280. (doi:10.1128/МСB.24.12.52695280.2004)

Migeon CJ, Tyler FH, Mahoney JP, Florentin AA, Castle H, Bliss EL \& Samuels LT 1956 The diurnal variation of plasma levels and urinary excretion on 17-hydroxycorticosteroids in normal subjects, night workers and blind subjects. Journal of Clinical Endocrinology and Metabolism 16 622-633. (doi:10.1210/jcem-16-5-622)

Miyazaki K, Itoh N, Ohyama S, Kadota K \& Oishi K 2013 Continuous exposure to a novel stressor based on water aversion induces abnormal circadian locomotor rhythms and sleep-wake cycles in mice. PLOS ONE 8 e55452. (doi:10.1371/journal.pone.0055452)

Mizoguchi K, Ishige A, Takeda S, Aburada M \& Tabira T 2004 Endogenous glucocorticoids are essential for maintaining prefrontal cortical cognitive function. Journal of Neuroscience 24 5492-5499. (doi:10.1523/ JNEUROSCI.0086-04.2004)

Mizoguchi K, Ikeda R, Shoji H, Tanaka Y \& Tabira T 2008 Suppression of glucocorticoid secretion induces a behaviorally depressive state in rotarod performance in rat. Pharmacology, Biochemistry, and Behavior 90 730-734. (doi:10.1016/j.pbb.2008.05.021)

Moore RY \& Eichler VB 1972 Loss of a circadian adrenal corticosterone rhythm following suprachiasmatic lesions in the rat. Brain Research $\mathbf{4 2}$ 201-206. (doi:10.1016/0006-8993(72)90054-6)

Morimoto M, Morita N, Ozawa H, Yokoyama K \& Kawata M 1996 Distribution of glucocorticoid receptor immunoreactivity and mRNA in the rat brain: an immunohistochemical and in situ hybridization study. Neuroscience Research 26 235-269. (doi:10.1016/S01680102(96)01105-4)

Morsink MC, Steenbergen PJ, Vos JB, Karst H, Joëls M, De Kloet ER \& Datson NA 2006 Acute activation of hippocampal glucocorticoid receptors results in different waves of gene expression throughout time. Journal of Neuroendocrinology 18 239-252. (doi:10.1111/j.1365-2826. 2006.01413.x)

Mukai H, Hatanaka Y, Mitsuhashi K, Hojo Y, Komatsuzaki Y, Sato R, Murakami G, Kimoto T \& Kawato S 2011 Automated analysis of spines from confocal laser microscopy images: application to the discrimination of androgen and estrogen effects on spinogenesis. Cerebral Cortex 21 2704-2711. (doi:10.1093/cercor/bhr059)

ffrench-Mullen JM 1995 Cortisol inhibition of calcium currents in guinea pig hippocampal CA1 neurons via G-protein-coupled activation of protein kinase C. Journal of Neuroscience 15 903-911.

Ooishi Y, Mukai H, Hojo Y, Murakami G, Hasegawa Y, Shindo T, Morrison JH, Kimoto T \& Kawato S 2012 Estradiol rapidly rescues synaptic transmission from corticosterone-induced suppression via synaptic/extranuclear steroid receptors in the hippocampus. Cerebral Cortex 22 926-936. (doi:10.1093/cercor/bhr164)

Perez-Cruz C, Simon M, Flügge G, Fuchs E \& Czéh B 2009 Diurnal rhythm and stress regulate dendritic architecture and spine density of pyramidal neurons in the rat infralimbic cortex. Behavioural Brain research 205 406-413. (doi:10.1016/j.bbr.2009.07.021)

Pilpel Y \& Segal M 2004 Activation of PKC induces rapid morphological plasticity in dendrites of hippocampal neurons via Rac and Rhodependent mechanisms. European Journal of Neuroscience 19 3151-3164. (doi:10.1111/j.0953-816X.2004.03380.x)

Qian X, Droste SK, Lightman SL, Reul JM \& Linthorst AC 2012 Circadian and ultradian rhythms of free glucocorticoid hormone are highly synchronized between the blood, the subcutaneous tissue, and the brain. Endocrinology 153 4346-4353. (doi:10.1210/en.2012-1484)

Reul JM \& de Kloet ER 1985 Two receptor systems for corticosterone in rat brain: microdistribution and differential occupation. Endocrinology 117 2505-2511. (doi:10.1210/endo-117-6-2505)

Rimmele U, Meier F, Lange T \& Born J 2010 Suppressing the morning rise in cortisol impairs free recall. Learning \& Memory 17 186-190. (doi:10.1101/lm.1728510)

Roberson ED, English JD, Adams JP, Selcher JC, Kondratick C \& Sweatt JD 1999 The mitogen-activated protein kinase cascade couples PKA and 
PKC to cAMP response element binding protein phosphorylation in area CA1 of hippocampus. Journal of Neuroscience 19 4337-4348.

Roozendaal B, Bohus B \& McGaugh JL 1996 Dose-dependent suppression of adrenocortical activity with metyrapone: effects on emotion and memory. Psychoneuroendocrinology 21 681-693. (doi:10.1016/S03064530(96)00028-5)

Roozendaal B, Quirarte GL \& McGaugh JL 2002 Glucocorticoids interact with the basolateral amygdala $\beta$-adrenoceptor - cAMP/cAMP/PKA system in influencing memory consolidation. European Journal of Neuroscience 15 553-560. (doi:10.1046/j.0953-816x.2001.01876.x)

Ruby NF, Hwang CE, Wessells C, Fernandez F, Zhang P, Sapolsky R \& Heller HC 2008 Hippocampal-dependent learning requires a functional circadian system. PNAS 105 15593-15598. (doi:10.1073/pnas. 0808259105)

Sapolsky RM, Krey LC \& McEwen BS 1984 Glucocorticoid-sensitive hippocampal neurons are involved in terminating the adrenocortical stress response. PNAS 81 6174-6177. (doi:10.1073/pnas.81.19.6174)

Scott RW, Hooper S, Crighton D, Li A, König I, Munro J, Trivier E, Wickman G, Morin P, Croft DR et al. 2010 LIM kinases are required for invasive path generation by tumor and tumor-associated stromal cells. Journal of Cell Biology 191 169-185. (doi:10.1083/jcb.201002041)

Shi Y, Pontrello CG, DeFea KA, Reichardt LF \& Ethell IM 2009 Focal adhesion kinase acts downstream of EphB receptors to maintain mature dendritic spines by regulating cofilin activity. Journal of Neuroscience $\mathbf{2 9}$ 8129-8142. (doi:10.1523/JNEUROSCI.4681-08.2009)

Venugopal SK, Chen J, Zhang Y, Clemens D, Follenzi A \& Zern MA 2007 Role of MAPK phosphatase-1 in sustained activation of JNK during ethanol-induced apoptosis in hepatocyte-like VL-17A cells. Journal of Biological Chemistry 282 31900-31908. (doi:10.1074/jbc.M703729200)

Vrang N, Larsen PJ \& Mikkelsen JD 1995 Direct projection from the suprachiasmatic nucleus to hypophysiotrophic corticotropin-releasing factor immunoreactive cells in the paraventricular nucleus of the hypothalamus demonstrated by means of Phaseolus vulgaris-leucoagglutinin tract tracing. Brain Research 684 61-69. (doi:10.1016/00068993(95)00425-P)

Weaver AM, Karginov AV, Kinley AW, Weed SA, Li Y, Parsons JT \& Cooper JA 2001 Cortactin promotes and stabilizes Arp2/3-induced actin filament network formation. Current Biology 11 370-374. (doi:10.1016/S0960-9822(01)00098-7)

Wiegert O, Joëls M \& Krugers H 2006 Timing is essential for rapid effects of corticosterone on synaptic potentiation in the mouse hippocampus. Learning \& Memory 13 110-113. (doi:10.1101/1m.87706)

Woolley CS, Gould E \& McEwen BS 1990 Exposure to excess glucocorticoids alters dendritic morphology of adult hippocampal pyramidal neurons. Brain Research 531 225-231. (doi:10.1016/00068993(90)90778-A)

Yang G \& Gan W-B 2012 Sleep contributes to dendritic spine formation and elimination in the developing mouse somatosensory cortex. Developmental Neurobiology 72 1391-1398. (doi:10.1002/dneu.20996)

Yang N, Higuchi O, Ohashi K, Nagata K, Wada A, Kangawa K, Nishida E \& Mizuno K 1998 Cofilin phosphorylation by LIM-kinase 1 and its role in Rac-mediated actin reorganization. Nature 393 809-812. (doi:10.1038/ 31735)

Yoshiya M, Komatsuzaki Y, Hojo Y, Ikeda M, Mukai H, Hatanaka Y, Murakami G, Kawata M, Kimoto T \& Kawato S 2013 Corticosterone rapidly increases thorns of CA3 neurons via synaptic/extranuclear glucocorticoid receptor in rat hippocampus. Frontiers in Neural Circuits 7 191. (doi:10.3389/fncir.2013.00191)

Yuen GS, McEwen BS \& Akama KT 2011 LIM kinase mediates estrogen action on the actin depolymerization factor Cofilin. Brain Research 1379 44-52. (doi:10.1016/j.brainres.2010.07.067)

Received in final form 14 May 2015

Accepted 1 June 2015

Accepted Preprint published online 1 June 2015
(C) 2015 Society for Endocrinology Printed in Great Britain 